\title{
Type A behavior and normal habitual sleep duration
}

\author{
ROBERT A. HICKS, ROBERT J. PELLEGRINI, SHARON MARTIN, \\ LINDA GARBESI, DARLYNE ELLIOTT, and JAMES HAWKINS \\ San Jose State University, San Jose, California 95192
}

\begin{abstract}
The responses of 361 college students to the Jenkins Activity Survey and a self-report sleep questionnaire were used to demonstrate an inverse relationship between normal habitual sleep duration and level of Type A behavior. The possibility that patterns of sleep may be implicated in the development of Type A behavior in some individuals was considered.
\end{abstract}

This paper reports a correlation between response to the Jenkins Activity Survey (JAS) and self-reported normal habitual sleep duration that may provide further insight into the processes underlying the development of Type A behavior. Our research was the outgrowth of an attempt to resolve an apparent conflict between the results of two sets of studies (Hartmann, Baekeland, \& Zwilling, 1972; Webb \& Friel, 1971) in which the relationships between extremes in normal habitual sleep duration (i.e., short and long sleepers) and certain aspects of personality had been correlated. To elaborate, Webb and Friel attempted to prove the null hypothesis for personality differences between short and long sleepers, with "personality" defined by a battery of tests that included the Minnesota Multiphasic Personality Inventory, the California Personality Inventory, the Zung Depression Scale, and the Cornell Medical Index.

While, in their first study, Hartmann et al. (1972) did find differences between short and long sleepers on some of the subtests of the scales used by Webb and Friel (1971), that aspect of their data was not replicated in subsequent research (Spinweber \& Hartmann, 1976). However, in the aforementioned studies, Hartmann and his colleagues collected both interview and clinical behavioral rating data from their short and long sleepers. With those measures (i.e., with data not considered by Webb and Friel), Hartmann et al. found, and replicated, a fairly pronounced pattern of personality differences between short and long sleepers. Essentially, Hartmann et al. (1972) described the short sleepers as efficient, energetic, ambitious, but "preprogrammed," nonworriers who tended to "avoid problems by keeping busy and by denial which in

We are grateful to David C. Glass for providing a copy of the JAS and the scoring key, to Signe Gary for her help in preparing the manuscript, and to the San Jose State University Foundation for providing funds for this research. Reprints may be obtained from Robert A. Hicks, Department of Psychology, San Jose State University, San Jose, California 95192. some cases approached hypomania" (p. 467). On the other hand, the long sleepers were seen as less aggressive and more reflective individuals who were constantly "reprogramming" themselves.

In view of the data base for the characterizations of short and long sleepers, one could argue that the Hartmann et al. conclusions were tainted, to an unknown degree, by observer bias effects. Thus, the Hartmann et al. descriptions of the behavior of short and long sleepers should be cross-validated against more objective scales. In this regard, we were impressed by the similarity between the behavior of the Hartmann et al. short sleepers and the Friedman and Roseman (1977) behavioral composite of the coronary-prone Type A man (also, see Jenkins, 1978). Therefore, an adaptation of the JAS for college students (Glass, 1977) is well suited for the purpose of testing the validity of the Hartmann et al. clinical observations. (It should be noted that the JAS is the psychometric measure that is most commonly used to define the Type A/Type B dichotomy.) Specifically, we predicted that normal habitual sleep duration and score on the JAS would be inversely related. That is, the shorter the sleep duration, the greater the level of Type A behavior.

\section{METHOD}

To test the hypothesis, more than 500 college students were given the JAS and a self-report sleep questionnaire (Hicks \& Pellegrini, 1977). To assess normal habitual sleep duration, the following questions were asked: "How many hours do you sleep each night?" "How long have you been experiencing this duration of sleep?" "Are you satisfied with the amount of sleep you get?" We excluded from further consideration individuals who reported a variable sleep pattern, who had experienced a given duration for less than 1 year, or who were dissatisfied with their sleep. (For a discussion that supports the need to control these parameters, see Cartwright, 1978.) As a result of the imposition of these selection criteria, the responses of 361 students provided the data base for the study. The subjects were subdivided into five sleep duration groups. The sleep duration intervals that define the groups, together with the number of students in each group, are shown in Table 1. The JAS was scored using the key developed by Glass (1977) for college students. 
Table 1

Means and Standard Deviations on the JAS for the Five Sleep Duration Groups

\begin{tabular}{lrrrrr}
\hline & \multicolumn{5}{c}{ Hours of Sleep Per Night } \\
\cline { 2 - 6 } Variable & $<6$ & 6 to 7 & 7 to 8 & 8 to 9 & $>9$ \\
\hline N & 18 & 65 & 140 & 101 & 37 \\
Mean & 10.6 & 9.2 & 8.6 & 7.9 & 7.2 \\
SD & 4.1 & 3.6 & 3.2 & 3.1 & 3.1 \\
\hline
\end{tabular}

\section{RESULTS AND DISCUSSION}

The mean JAS score and the standard deviation for each sleep duration group are given in Table 1. A oneway analysis of variance testing the differences between the means revealed that sleep duration and JAS score were significantly related $[\mathrm{F}(4,356)=4.91, \mathrm{p}<.01]$. Therefore, the pattern of the means shown in Table 1 conformed to the predicted inverse relationship between sleep duration and level of Type A behavior. In addition to providing a limited psychometric validation of the Hartmann et al. (1972) clinical observations, these data may provide some insight into a mechanism which may assist some individuals in the development of Type A behavior. The following paragraphs are a brief elaboration of that hypothesis.

It has been demonstrated that, while the amount of deep NREM sleep (i.e., Stages 3 and 4) remains relatively constrained over the range of habitual sleep durations, the amount of REM sleep is directly proportional to the total habitual sleep duration (e.g., Cartwright, 1978). Hence, it seems plausible to assume that short sleepers are, to a moderate degree, chronically REM deprived. The behavioral changes that have been observed as a consequence of REM deprivation may, to a degree, also be characteristic of habitual short sleepers. In this regard, Vogel (1975), in a recent review, suggested that the outstanding consequence of REM deprivation is an increase in general energy (or drive) level. Further, it has been reported (Hicks, Pellegrini, Hawkins, \& Moore, 1978) that, for college students, shortened sleep seems to be a relatively recently acquired sleep habit. With these points in mind, consider the following hypothetical sequel in the formation of Type A behavior. First, an individual finds himself in a high-demand situation, for example, entry into college. To achieve he works longer and, as a consequence, sleeps less. He finds that, contrary to expectations, less sleep makes him feel more energetic and thus is reinforcing. As the hypothetical cycle continues, the person experiences both more time to work and a greater level of energy with which to work; thus, he is better able to achieve in the situation that originally initiated the sequence of events.
Finally, it seems fairly well documented (e.g., Glass, 1977) that Type A individuals are less able to cope with stress and that their inability to cope provides an explanation for the greater incidence of coronary disease that Type A individuals tend to display. With respect to our sleep duration/Type A hypothesis, recent discussion and research (e.g., Cartwright, 1978; Glaubman, Orbach, Aviram, Frieder, Frieman, Pelled, \& Glaubman, 1978; Greenberg, 1977; Hicks \& Sawrey, 1978) have noted that REM deprivation (i.e., a possible consequence of short sleep) seems to heighten susceptibility to stressors and/or to reduce an individual's ability to cope with stressful situations. Thus, it may prove to be the case that the stress proneness of some Type A individuals is in part mediated by acquired patterns of shortened sleep.

To conclude, though the hypothesis advanced in this paper may be of a tenuous nature, the available evidence is compelling. Certainly, the hypothesis merits additional testing.

\section{REFERENCES}

Cartwright, R. D. A primer on sleep and dreaming. Reading, Mass: Addison-Wesley, 1978.

Friedman, M., \& Rosenman, R. H. The key cause: Type A behavior pattern. In A. Monat \& R. S. Lazarus (Eds.), Stress and coping. New York: Columbia University Press, 1977.

Glass, D. C. Behavior patterns, stress, and coronary disease. Hillsdale, N.J: Erlbaum, 1977.

Glaubman, H., Orbach, I., Aviram, O., Frieder, I., Frieman, M., Pelled, O., \& Glaubman, R. REM deprivation and divergent thinking. Psychophysiology, 1978, 15, 75-79.

GREENBERG, R. On understanding sleep disorders and their psychopathology. McLean Hospital Journal, 1977, 2, 139-146.

Hartmann, E., Baekeland, F., \& Zwilling, G. R. Psychological differences between long and short sleepers. Archives of general psychiatry, 1972, 26, 463-468.

Hicks, R. A., \& Pellegrini, R. J. Anxiety levels of short and long sleepers. Psychological Reports, 1977, 41, 569-570.

Hicks, R. A., Pellegrini, R. J., Hawkins, J., \& Moore, J. D. Self-reported consistency of normal habitual sleep durations of college students. Perceptual and Motor Skills, 1978, 47, 457-458.

Hicks, R. A., \& SAwrey, J. M. REM sleep deprivation and stress susceptibility in rats. Psychological Record, 1978, 28, 187-191.

JENKINS, C. D. Behavioral risk factors in coronary artery disease. In W. P. Creger, C. H. Coggins, \& E. W. Hancock (Eds.), Annual review of medicine: Selected topics in the clinical sciences (Vol. 29). Palo Alto: Annual Reviews, 1978.

Spinweber, C. L., \& Hartmann, E. Long and short sleepers: Male and female subjects' sleep, personality and biochemical measures. Sleep Research, 1976, 5, 112.

Vogel, G. W. A review of REM sleep deprivation. Archives of General Psychiatry, 1975, 32, 749-761.

WebB, W. B., \& FrieL, J. Sleep stage and personality characteristics of "natural" long and short sleepers. Science, 1971, 171, 587-588.

(Received for publication June 4, 1979.) 